OPEN ACCESS

Check for updates

\section{Use of personal protective equipment against coronavirus disease 2019 by healthcare professionals in Wuhan, China: cross sectional study}

\author{
Min Liu, ${ }^{1}$ Shou-Zhen Cheng, ${ }^{1}$ Ke-Wei Xu, ${ }^{2}$ Yang Yang,,${ }^{3}$ Qing-Tang Zhu, ${ }^{1}$ Hui Zhang, ${ }^{1}$ Da-Ya Yang, \\ Shu-Yuan Cheng, ${ }^{1}$ Han Xiao, ${ }^{1}$ Ji-Wen Wang, ${ }^{2}$ He-Rui Yao, ${ }^{2}$ Yu-Tian Cong, ${ }^{3}$ Yu-Qi Zhou, ${ }^{3}$ Sui Peng, ${ }^{1}$ \\ Ming Kuang, ${ }^{1}$ Fan-Fan Hou, ${ }^{4}$ KK Cheng, ${ }^{5}$ Hai-Peng Xiao ${ }^{1}$
}

${ }^{1}$ The First Affiliated Hospital of Sun Yat-sen University, Guangzhou, 510080, China

${ }^{2}$ Sun Yat-sen Memorial Hospital, Sun Yat-sen University,

${ }^{3}$ The Third Affiliated Hospital of Sun Yat-sen University, Guangzhou, China Medical University, Guangzhou, China

Institute of Applied Health Birmingham, Birmingham, UK

Correspondence to: H-P Xiao xiaohp@mail.sysu.edu.cn (ORCID 0000-0002-4188-336X) Additional material is published online only. To view please visit the journal online.

Cite this as: BMJ 2020;369:m2195 http://dx.doi.org/10.1136/bmj.m2195

Accepted: 2 June 2020 Guangzhou, China

${ }^{4}$ Nan Fang Hospital, Southern Research, University of

\section{ABSTRACT}

OBJECTIVE

To examine the protective effects of appropriate personal protective equipment for frontline healthcare professionals who provided care for patients with

\section{DESIGN}

Cross sectional study.

SETTING

Four hospitals in Wuhan, China.

\section{PARTICIPANTS}

420 healthcare professionals ( 116 doctors and 304 nurses) who were deployed to Wuhan by two affiliated hospitals of Sun Yat-sen University and Nanfang Hospital of Southern Medical University for 6-8 weeks from 24 January to 7 April 2020. These study participants were provided with appropriate personal protective equipment to deliver healthcare to patients admitted to hospital with covid-19 and were involved in aerosol generating procedures. 77 healthcare professionals with no exposure history to covid-19 and 80 patients who had recovered from covid-19 were recruited to verify the accuracy of antibody testing.

\section{MAIN OUTCOME MEASURES}

Covid-19 related symptoms (fever, cough, and dyspnoea) and evidence of severe acute respiratory syndrome coronavirus 2 (SARS-CoV-2) infection, defined as a positive test for virus specific nucleic acids in nasopharyngeal swabs, or a positive test for IgM or IgG antibodies in the serum samples. coronavirus disease 2019 (covid-19).

\section{WHAT IS ALREADY KNOWN ON THIS TOPIC}

Severe acute respiratory syndrome coronavirus 2 (SARS-CoV-2) infection among healthcare professionals was very common in Wuhan and in other countries in the early phase of the outbreak

Inappropriate personal protective equipment is believed to be a major factor in the prevalence of SARS-CoV-2 infection among healthcare professionals

The epidemiology and serological response of healthcare professionals with high exposure to SARS-CoV-2 is not known

\section{WHAT THIS PAPER ADDS}

Appropriate personal protective equipment gives healthcare professionals a high level of protection against coronavirus disease 2019 (covid-19)

Despite a high risk of exposure, no signs of infection or serological response were detected in any of the study participants who were deployed to Wuhan to care for patients with covid-19

\section{RESULTS}

The average age of study participants was 35.8 years and $68.1 \%(286 / 420)$ were women. These study participants worked 4-6 hour shifts for an average of 5.4 days a week; they worked an average of 16.2 hours each week in intensive care units. All 420 study participants had direct contact with patients with covid-19 and performed at least one aerosol generating procedure. During the deployment period in Wuhan, none of the study participants reported covid-19 related symptoms. When the participants returned home, they all tested negative for SARSCoV-2 specific nucleic acids and IgM or IgG antibodies (95\% confidence interval 0.0 to $0.7 \%$ ).

\section{CONCLUSION}

Before a safe and effective vaccine becomes available, healthcare professionals remain susceptible to covid-19. Despite being at high risk of exposure, study participants were appropriately protected and did not contract infection or develop protective immunity against SARS-CoV-2. Healthcare systems must give priority to the procurement and distribution of personal protective equipment, and provide adequate training to healthcare professionals in its use.

\section{Introduction}

The coronavirus disease 2019 (covid-19) pandemic has now spread to more than 200 countries. ${ }^{1}$ In the early phase of the outbreak in Wuhan, China, the healthcare system was overwhelmed as the number of patients with severe disease surged and many healthcare professionals were infected. ${ }^{2}$ Similar problems occurred in Europe and the United States. ${ }^{3-5}$ During an epidemic, the infection of healthcare professionals has a major negative impact on the capacity to treat patients, on the morale of professionals, and on public confidence. Therefore, appropriate protection of healthcare professionals is of utmost importance in the response to covid-19 in any healthcare system.

During the early phase of the outbreak it was unclear what personal protective equipment would provide sufficient protection to healthcare professionals when caring for patients with covid-19. A clinical trial had shown that N95 respirators are superior to medical masks in preventing respiratory virus infection in healthcare workers. ${ }^{6}$ A meta-analysis reported that regular hand hygiene provided a significant protective effect and facemask use was associated with a nonsignificant protective effect against influenza infection during the 2009 pandemic. ${ }^{7}$ Whether these findings 
would apply to severe acute respiratory syndrome coronavirus 2 (SARS-CoV-2) infection was unknown.

When the epidemic in Wuhan accelerated at the end of January 2020, healthcare professionals from other provinces and municipal cities were deployed to Wuhan. ${ }^{8}$ These healthcare professionals worked on the frontline and had direct contact with patients with covid-19 for 6-8 weeks. Most were exposed to patients with severe disease in intensive care units where they performed aerosol generating procedures, and the risk of infection was probably high. These relief teams were equipped with relatively abundant and appropriate personal protective equipment, and so this study examined whether such provision protects healthcare professionals from SARS-CoV-2 infection.

\section{Methods}

\section{Study design, setting, and participants}

In this cross sectional study, we recruited 420 healthcare professionals from the First Affiliated Hospital and Sun Yat-sen Memorial Hospital (both of Sun Yat-sen University), and Nanfang Hospital of Southern Medical University who were deployed to Wuhan from 24 January to 7 April 2020. All of these healthcare professionals worked on the frontline for 6-8 weeks caring for patients with covid-19. To assess the validity of serological testing, blood samples from 77 healthy healthcare professionals with no symptoms and no history of SARS-CoV-2 infection were collected at the home hospitals as negative controls. We used blood samples from 80 age and sex matched patients who had recovered from covid-19 as positive controls. Informed consent was obtained from all participants. The study was approved by the Research Ethics Commissions of the First Affiliated Hospital and Sun Yat-sen Memorial Hospital of Sun Yat-sen University, and Nanfang Hospital of Southern Medical University.

The 420 healthcare professionals consisted of doctors and nurses, and $67.6 \%$ were from departments other than intensive care, respiratory medicine, and infectious diseases. These healthcare professionals were deployed to four hospitals in Wuhan: West division of Union Hospital, Guanggu division of Tongji Hospital, Hankou Hospital, and Honghu Hospital. The participants underwent daily monitoring for covid-19 related symptoms, including fever, cough, and dyspnoea, during their stay in Wuhan. The temperature of participants was measured twice a day with a digital infrared thermometer, and participants reported any symptoms of cough and dyspnoea twice a day.

\section{Infection control measures}

Healthcare professionals who were involved in the direct care of patients with covid-19 were equipped with standardised personal protective equipment, including protective suits, masks, gloves, goggles, face shields, and gowns. These healthcare professionals received training in the correct use of personal protective equipment and in reducing their exposure to infection when caring for patients with covid-19. The practice protocol also included working in pairs to mutually observe the putting on and taking off of personal protective equipment. When taking off personal protective equipment, healthcare professionals followed the outside-in rule, meaning that the outermost layer of personal protective equipment would be taken off first. Hand sanitiser was used after each step. Videos showing details of putting on and taking off personal protective equipment can be accessed online (putting on personal protective equipment: http://en.gzsums. net/uploadfiles/2020/03/03-23/4.mp4; taking off personal protective equipment: http://en.gzsums.net/ uploadfiles/2020/03/03-23/5.mp4).

Several other measures were adopted for infection control: minimising direct contact with patients; sanitising gloves after treating each patient; replacing gloves promptly when contaminated; hand washing when changing gloves; maintaining at least one metre distance between healthcare professionals or healthcare professionals and patients if possible; avoiding touching their own heads and faces; and strictly following the hand washing procedure as recommended by the World Health Organization. ${ }^{9}$

Participants lived in hotels designated for frontline healthcare professionals and special shuttles were used for transportation. After work, participants followed the rules of social distancing and social interaction was minimised. Every healthcare professional wore a mask in public places and when on the shuttles. All healthcare professionals had their meals in their own rooms and all food was delivered.

\section{Data collection}

We used an online questionnaire to collect data on clinical practice, which included the frequency of being involved in aerosol generating procedures. These procedures included tracheal intubation, invasive and non-invasive mechanical ventilation, gastric intubation, sputum aspiration, aerosol inhalation, tracheostomy, tracheostomy care, and throat swab collection. Line managers made phone calls and sent emails to healthcare professionals who had not completed the questionnaire to increase the response rate. The questionnaire consisted of objective questions related to daily clinical practice in following the protocol for standard personal protective equipment to reduce recall bias.

\section{Study procedures}

Nasopharyngeal swabs were collected three times from each participant during the two week quarantine period after they had returned home from Wuhan: on day 1, day 7, and day 14. Local Center for Disease Control and Prevention testing laboratories performed SARS-CoV-2 nucleic acid testing by using reverse transcriptase polymerase chain reaction. Sample collection and laboratory testing procedures were carried out in accordance with WHO guidance. ${ }^{10}$ Blood samples were collected from participants at the end of the two week quarantine period. Blood samples 
were also obtained from healthcare professionals without covid-19 exposure and from patients who had recovered from covid-19 as negative and positive controls, respectively. Serum samples were used to test for IgM and IgG antibodies against spike protein $\mathrm{S} 1$ and nucleocapsid (N) protein of SARS-CoV-2 by chemiluminescence immunoassay using a commercial kit (CLIA assays kit, YHLO). ${ }^{11}$ The sensitivity and specificity of the assay for IgM are $88.2 \%$ and $99.0 \%$, respectively; the sensitivity and specificity for IgG are $97.8 \%$ and $97.9 \%$, respectively. These results were reported by the manufacturer, and were independently validated. ${ }^{11}$ Testing was performed by well trained specialists with over three years' experience who followed strict protocols. All of the planned tests were completed with no invalid or missing data.

\section{Statistical analysis}

IgM and IgG data are presented as means and standard deviations. We performed statistical analyses by using Prism 8.0 software. Comparisons were assessed using Kruskal-Wallis test measurements and multiple comparison. We analysed the $95 \%$ confidence intervals of infection rates by Wilson score interval. A two sided $P$ value less than 0.05 was considered significant.

\section{Patient and public involvement}

Because of the rapid global progression of covid-19, there was a need to disseminate information quickly and thus patients were not directly involved in the development, implementation, or interpretation of this study. This study was based on questionnaires and laboratory tests, which were not accessible to nonmedical or non-research personnel.

\section{Results}

\section{Participants and exposure}

The 420 healthcare professionals included in this study consisted of 116 doctors and 304 nurses. The average age of participants was 35.8 years; 286 were women $(68.1 \%)$ and 134 were men $(31.9 \%$; table 1). During practice, participants were equipped with standardised personal protective equipment, including protective suits, masks, gloves, goggles, face shields, and gowns (table 2).

All participants worked in four frontline hospitals in Wuhan and provided care for patients with severe or critical covid-19. Over $80 \%$ of these patients required critical care, and $10-15 \%$ needed mechanical ventilation. Participants worked 4-6 hour shifts for an average of 5.4 days a week; they worked an average of 16.2 hours each week in intensive care units. All healthcare professionals had performed at least one aerosol generating procedure. These procedures included tracheal intubation, noninvasive mechanical ventilation, gastric intubation, sputum aspiration, aerosol inhalation, tracheostomy, tracheostomy care, and throat swab collection (table 1 and table 3). None of the participants reported fever, cough, or dyspnoea during their 6-8 week deployment to Wuhan.

\section{SARS-CoV-2 nucleic acid and specific IgM or IgG testing}

None of the nasopharyngeal swabs collected from the participants tested positive for nucleic acids on the reverse transcriptase polymerase chain reaction assay for SARS-CoV-2 (table 1). None of the serum samples of participants tested positive for SARS-CoV-2 specific IgM or IgG antibodies (95\% confidence interval 0.0 to $0.7 \%$ ). No serological response to SARS-CoV-2 was detected in the 77 control healthcare professionals with no history of exposure to covid-19 (fig 1). All 80 serum samples from patients who had recovered from covid-19 had high titres of either SARS-CoV-2 IgM or IgG (fig 1).

\section{Discussion}

Our study investigated whether appropriate personal protective equipment can protect frontline healthcare professionals who are exposed to SARS-CoV-2 infection. Despite a high risk of exposure, no evidence was found of infection in any of the 420 participants as shown by negative test results for nucleic acids and specific IgM or IgG antibodies.

A large number of healthcare professionals around the world have been infected with SARS-CoV-2. ${ }^{12}$ In one early report issued by the WHO-China Joint Mission, 3387 medical professionals in 476 hospitals had been infected with SARS-CoV-2. ${ }^{13}$ Another single centre study from Wuhan reported that of 138 patients in hospital with covid-19, 29\% were healthcare workers. ${ }^{14}$ Most of the affected healthcare professionals became infected in the early phase of the outbreak and the main reason was probably a lack of appropriate personal protective equipment. ${ }^{15}$

During the outbreak of severe acute respiratory syndrome in 2003, masks, gowns and hand washing were effective in reducing risk of infection, while healthcare professionals with less personal protective equipment had a higher incidence of infection. ${ }^{16}$ A recent study proposed that personal protective equipment appropriate for SARS-CoV-2 consists of protective masks, round caps, gloves, protective clothing, boot covers, and goggles or a face shield. By using this protection, the risk of infection among local healthcare professionals was considerably reduced, though not completely eliminated. ${ }^{17}$ During the early phase of the outbreak, little was known about SARSCoV-2. Reports suggested that the virus was primarily transmitted between people through respiratory droplets and contact routes, and that airborne transmission could occur in specific circumstances when procedures are performed that generate aerosols. ${ }^{18}$

In our study, all participants were responsible for the care of patients in hospital with severe covid-19, which included performing aerosol generating procedures on a routine basis. Therefore, we implemented a more stringent protocol for our participants as a necessary precaution, which included wearing N95 respirators and surgical masks at the same time (see supplementary table). In addition, participants were 


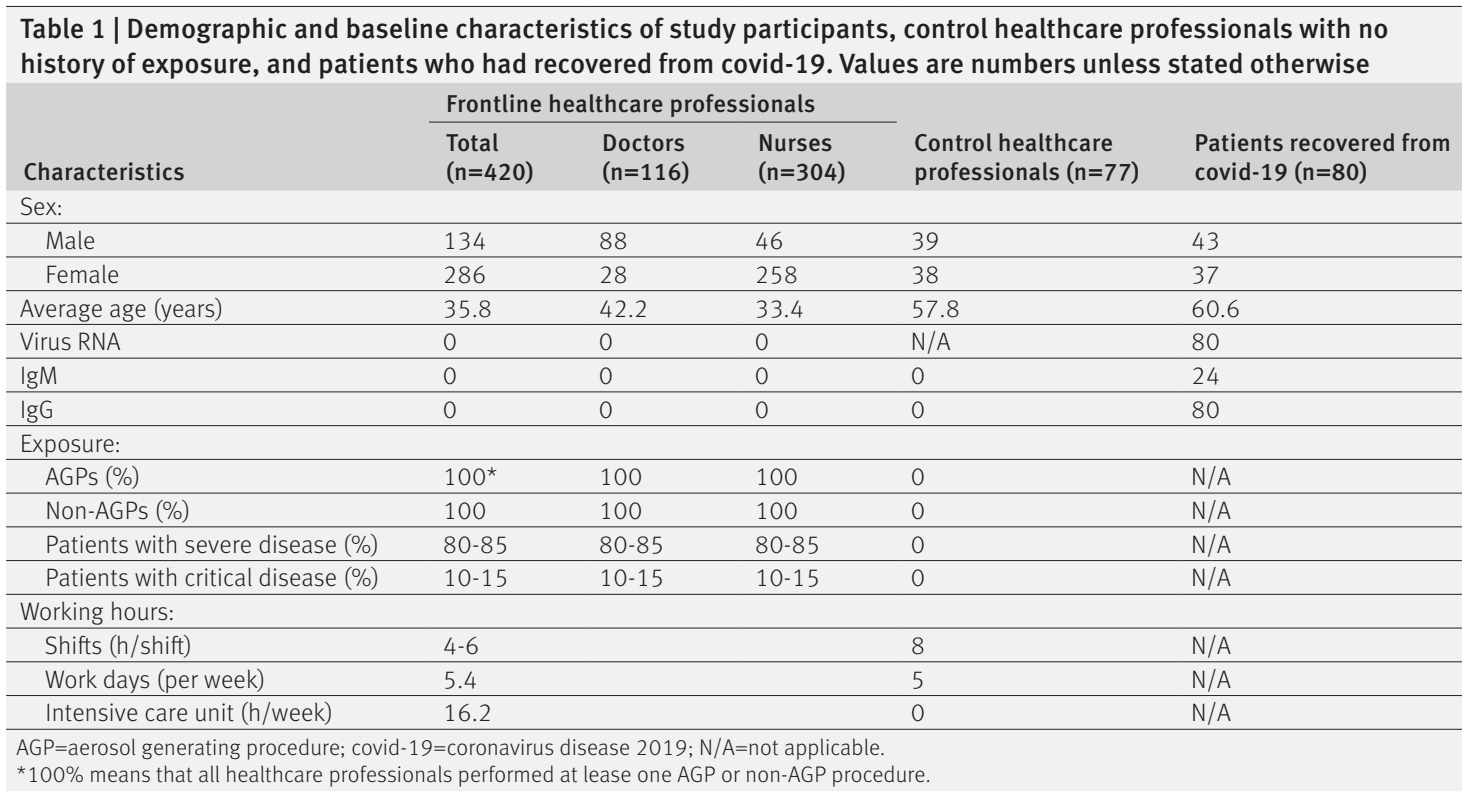

well trained in hand hygiene, putting on and taking off personal protective equipment, and performing aerosol generating procedures. Outside of work, participants stayed in designated hotels, followed strict rules including social distancing, and wore masks in public areas. Our results indicate that appropriate personal protective equipment in addition to adhering to standard recommendations had effectively protected our participants from SARS-CoV-2 infection in clinical settings with a high risk of exposure.

SARS-CoV-2 nucleic acid testing by using reverse transcriptase polymerase chain reaction is being used globally to diagnose covid-19. However, reports suggest that the false negative rate of nucleic acid testing could be as high as 21.4\%. ${ }^{19}{ }^{20}$ Antibodies against SARS-CoV-2 have been isolated from patients with covid-19, some of which have shown strong neutralising potency. ${ }^{21}$ Among all 420 participants in our study, none of the serum samples tested positive for IgM or IgG antibodies against SARS-CoV-2. Control healthcare professionals without exposure history also tested negative, but patients who had recovered from covid-19 tested positive. Although false negative tests have been reported for throat swab samples at various stages of the disease, ${ }^{22}$ a previous study showed detectable antibodies in 100\% of patients with covid-19 from day 15 after onset. ${ }^{23}$ Because serological tests were performed two weeks after our participants had returned from Wuhan, false negatives were unlikely. Our findings also suggest that these healthcare professionals did not acquire protective immunity and remained susceptible to the novel coronavirus.

\section{Limitations of this study}

Our study is limited in several ways. Firstly, the study is cross sectional in nature. Because the possibility of false negative results cannot be definitively excluded, future follow up is warranted. However, findings from future studies are unlikely to change our conclusion that healthcare professionals with appropriate personal protective equipment are highly protected. However, this study does not address the question of what the minimal level of personal protective equipment should be to effectively prevent infection among healthcare professionals. Secondly, because all the participants in our study worked in hospitals at the epicentre of the outbreak, caution should be given when these results are extrapolated to other settings. Also, our findings only apply to healthcare professionals who work on

\begin{tabular}{|c|c|c|c|c|c|}
\hline \multirow[b]{2}{*}{ Personal protective equipment } & \multicolumn{3}{|l|}{ Ward* } & \multicolumn{2}{|c|}{ AGP† exposure } \\
\hline & Intensive care units & Regular wards & No covid-19 contact area & AGPs & Non-AGPs \\
\hline \multicolumn{6}{|l|}{ Mask: } \\
\hline N95 respirator & + & + & - & + & + \\
\hline Surgical mask & + & + & + & + & + \\
\hline Medical suit & + & + & - & + & + \\
\hline Isolation gown & + & + & - & + & + \\
\hline Apron & - & - & - & + & - \\
\hline Gloves & + & + & - & + & + \\
\hline Eye protection & + & + & - & + & + \\
\hline Hair cover & + & + & - & + & + \\
\hline
\end{tabular}




\begin{tabular}{|c|c|c|c|c|c|c|c|c|}
\hline \multirow{2}{*}{$\begin{array}{l}\text { Aerosol generating } \\
\text { procedures }\end{array}$} & \multicolumn{4}{|c|}{ Cumulative procedures performed by doctors $(n=116)$} & \multicolumn{4}{|c|}{ Cumulative procedures performed by nurses $(n=304)$} \\
\hline & 0 & $<10$ & $10-20$ & $\$ 20$ & 0 & $<10$ & $10-20$ & 220 \\
\hline Tracheal intubation* & $53(45.7)$ & $43(37.1)$ & $14(12)$ & $6(5.2)$ & $304(100)$ & $0(0.0)$ & $0(0.0)$ & $0(0.0)$ \\
\hline Ventilation & $18(15.5)$ & $67(57.8)$ & $22(19)$ & $9(7.7)$ & $77(25.3)$ & $138(45.4)$ & $62(20.4)$ & $27(8.9)$ \\
\hline Gastric intubation & $116(100)$ & $0(0.0)$ & $0(0.0)$ & $0(0.0)$ & $156(51.3)$ & $141(46.4)$ & $7(2.3)$ & $0(0.0)$ \\
\hline Sputum aspiration & $116(100)$ & $0(0.0)$ & $0(0.0)$ & $0(0.0)$ & $0(0.0)$ & $61(20)$ & $127(41.8)$ & $116(38.2)$ \\
\hline Aerosol inhalation & $116(100)$ & $0(0.0)$ & $0(0.0)$ & $0(0.0)$ & $107(35.2)$ & $116(38.2)$ & $34(11.2)$ & $47(15.4)$ \\
\hline Oral care & $116(100)$ & $0(0.0)$ & $0(0.0)$ & $0(0.0)$ & $83(27.3)$ & $120(39.5)$ & $68(22.4)$ & $33(10.8)$ \\
\hline Tracheostomy care & $116(100)$ & $0(0.0)$ & $0(0.0)$ & $0(0.0)$ & $88(29)$ & $135(44.4)$ & $52(17.1)$ & $29(9.5))$ \\
\hline
\end{tabular}

the clinical frontline and are not generalisable to community settings.

Finally, the healthcare professionals in our study were working away from home and had limited social interactions after work. This probably contributed to the absence of infection. However, this limitation does not affect our conclusion that appropriate personal protective equipment is effective in preventing infection in healthcare professionals who work in highly exposed environments.

\section{Conclusion}

Before a safe and effective vaccine becomes available, healthcare professionals remain susceptible to covid-19. Despite being at high risk of exposure, healthcare professionals who were appropriately protected did not contract infection or develop protective immunity against SARS-COV-2. Healthcare systems must give priority to the procurement and distribution of personal protective equipment, and provide adequate training to healthcare professionals in its use.

We thank all patients involved in this study, as well as doctors, nurses, and researchers working together to fight against covid-19.

Contributors: ML, SZC, KWX, YY, QTZ, HZ, DYY, and SYC contributed equally to this paper. HPX and KKC conceived and designed the study. ML, SZC, KWX, YY, QTZ, JWW, HRY, YTC, YQZ, SP, and MK contributed to recruitment of patients and healthcare professionals, data collection, data analysis, and data interpretation. HZ, DYY, SYC, and HX contributed to literature search and data collection. HZ, DYY, SYC, $\mathrm{HX}$, and KKC drafted the manuscript. MK, FFH, KKC, and HPX are the guarantors. All authors approved the final version of the manuscript.
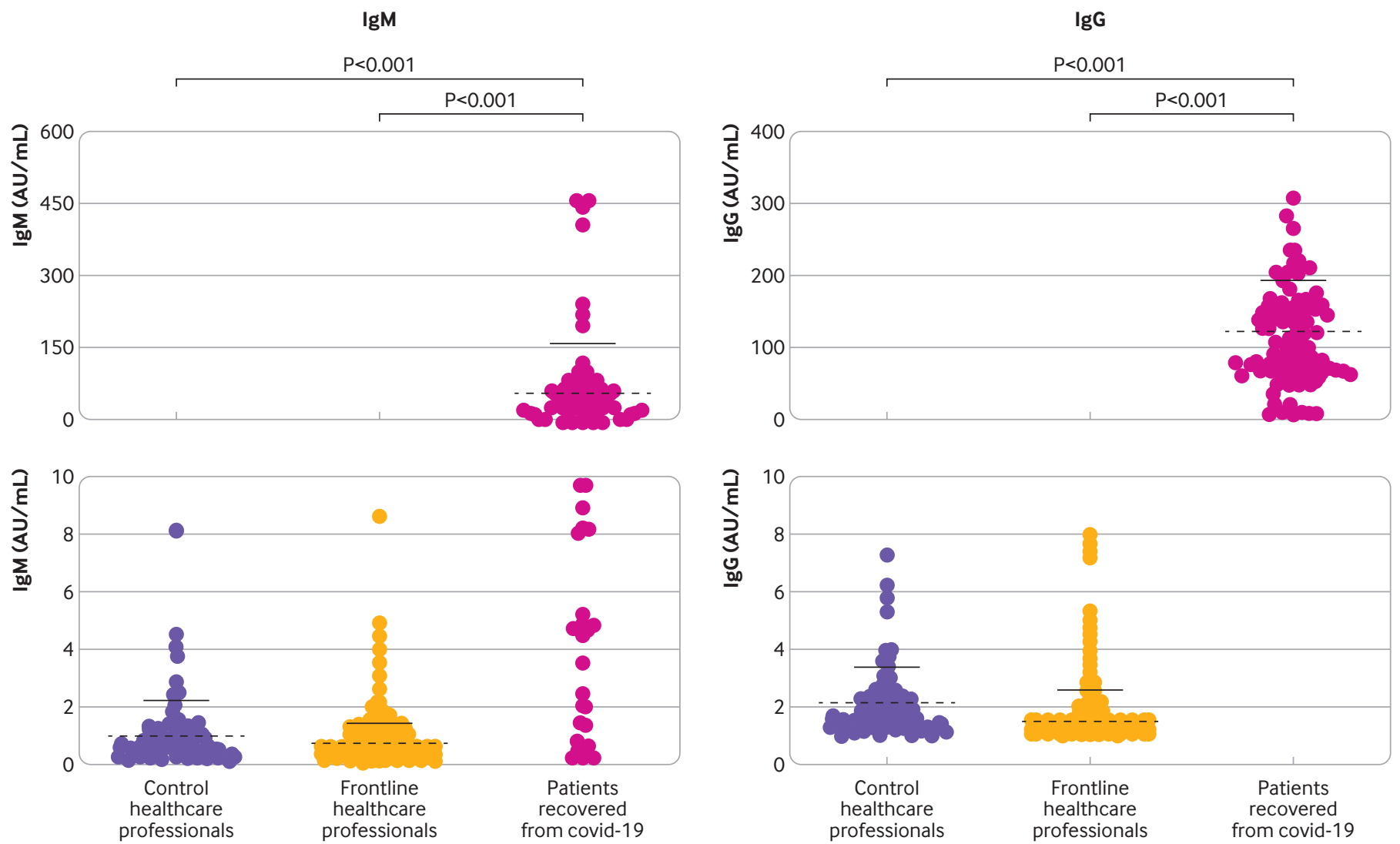

Fig 1 | Serological response to severe acute respiratory syndrome coronavirus 2 (SARS-CoV-2). Concentrations of SARS-CoV-2 specific IgM and IgG antibodies in serum samples of healthcare professionals who had been exposed to coronavirus disease 2019 (covid-19) in Wuhan were quantified by chemiluminescence immunoassay $(n=420)$. Serum samples from healthcare professionals without covid-19 exposure were used as negative controls $(n=77)$. Serum samples from patients who had recovered from covid-19 were used as positive controls $(n=80)$. Data are expressed as mean \pm standard deviation. Reference specified by manufacturer $(\ll 10 \mathrm{AU} / \mathrm{mL})$ 
The corresponding author attests that all listed authors meet authorship criteria and that no others meeting the criteria have been omitted.

Funding: This work was funded by grants from the First Affiliated Hospital of Sun Yat-sen University. The funder was not involved in the design and conduct of the study; collection, analysis, interpretation of data, writing of the report, or decision to submit the article for publication.

Competing interests: All authors have completed the ICMJE uniform disclosure form at www.icmje.org/coi disclosure.pdf and declare: grant funding from the First Affiliated Hospital of Sun Yat-sen University; no financial relationships with any organisations that might have an interest in the submitted work in the previous three years; no other relationships or activities that could appear to have influenced the submitted work.

Ethical approval: This study was approved by the First Affiliated Hospital of Sun Yat-sen University (2020-130). Written informed consent was obtained from all participants before enrolment.

Data sharing: No additional data available.

Dissemination to participants and related patient and public communities: The findings of this study will be disseminated to all clinical departments caring for patients with covid-19 at author affiliated institutions. In addition, our media relation departments will plan to further disseminate through press releases, as well as our institutional websites.

The manuscript's guarantors (MK, FFH, KKC, and HPX) affirm that the manuscript is an honest, accurate, and transparent account of the study being reported; that no important aspects of the study have been omitted; and that any discrepancies from the study as planned (and, if relevant, registered) have been explained.

This is an Open Access article distributed in accordance with the Creative Commons Attribution Non Commercial (CC BY-NC 4.0) license, which permits others to distribute, remix, adapt, build upon this work non-commercially, and license their derivative works on different terms, provided the original work is properly cited and the use is noncommercial. See: http://creativecommons.org/licenses/by-nc/4.0/.

1 Johns Hopkins University \& Medicine. Coronavirus COVID-19 Global Cases by the Center for Systems Science and Engineering (CSSE) at Johns Hopkins University (JHU). https://coronavirus.jhu.edu/map.html [cited on 14 April 2010].

2 Zhang Z, Liu S, Xiang M, et al. Protecting healthcare personnel from 2019-nCoV infection risks: lessons and suggestions. Front Med 2020;14:229-31. doi:10.1007/s11684-020-0765-x

3 Legido-Quigley H, Mateos-García JT, Campos VR, Gea-Sánchez M, Muntaner C, McKee M. The resilience of the Spanish health system against the COVID-19 pandemic. Lancet Public Health 2020;5:e251 2. doi:10.1016/S2468-2667(20)30060-8

4 Remuzzi A, Remuzzi G. COVID-19 and Italy: what next?Lancet 2020;395:1225-8. doi:10.1016/S0140-6736(20)30627-9

5 CDC COVID-19 Response Team. Characteristics of health care personnel with COVID-19 - United States, February 12-April 9, 2020. MMWR Morb Mortal Wkly Rep 2020;69:477-81.

6 Maclntyre CR, Wang Q, Cauchemez S, et al. A cluster randomized clinical trial comparing fit-tested and non-fit-tested N95 respirators to medical masks to prevent respiratory virus infection in health care workers. Influenza Other Respir Viruses 2011;5:170-9. doi:10.1111/ j.1750-2659.2011.00198.x

7 Saunders-Hastings P, Crispo JAG, Sikora L, Krewski D. Effectiveness of personal protective measures in reducing pandemic influenza transmission: a systematic review and meta-analysis. Epidemics 2017;20:1-20. doi:10.1016/j.epidem.2017.04.003

8 China NHC. Answering to the call, 42000+ HCPs threw down their gauntlets and joined the united fight against the deadly virus.: http://www.nhc.gov.cn/wjw/mtbd/202003/ e0d5f8a773b54fc39113988dbcb19136.shtml. [Accessed on 23 May 2020] [in Chinese]

9 World Health Organization. Clean hands protect against infection. https://www.who.int/gpsc/clean hands protection/en/ [Accessed on 20 May 2020]

10 World Health Organization. Laboratory testing for 2019 novel coronavirus (2019-nCoV) in suspected human cases: interim guidance 2020. https://www.who.int/publications-detail/ laboratory-testing-for-2019-novel-coronavirus-in-suspected-humancases-20200117 [Accessed on 4 February 2020]

11 Zeng H, Xu C, Fan J, et al. Antibodies in infants born to mothers with COVID-19 pneumonia. JAMA 2020. doi:10.1001/jama.2020.4861

12 Coronavirus disease 2019 (COVID-19) Situation Report-82. https://www.who.int/docs/default-source/coronaviruse/situationreports/20200411-sitrep-82-covid-19.pdf?sfvrsn=74a5d15_2 [Accessed on 21 May 2020]

13 WHO-China Joint Mission. Report of the WHO-China Joint Mission on Coronavirus Disease 2019 (COVID-19). https://www who.int/docs/ default-source/coronaviruse/who-china-joint-mission-on-covid-19final-report.pdf [Accessed on 24 May 2020]

14 Wang D, Hu B, Hu C, et al. Clinical characteristics of 138 hospitalized patients with 2019 novel coronavirus-infected pneumonia in Wuhan, China. JAMA 2020. doi:10.1001/jama.2020.1585

15 Wang J, Zhou M, Liu F. Reasons for healthcare workers becoming infected with novel coronavirus disease 2019 (COVID-19) in China. J Hosp Infect 2020;105:100-1. doi:10.1016/j. ihin.2020.03.002

16 Seto WH, Tsang D, Yung RW, et al, Advisors of Expert SARS group of Hospital Authority. Effectiveness of precautions against droplets and contact in prevention of nosocomial transmission of severe acute respiratory syndrome (SARS). Lancet 2003;361:1519-20. doi:10.1016/S0140-6736(03)13168-6

17 Ran L, Chen X, Wang Y, Wu W, Zhang L, Tan X. Risk factors of healthcare workers with corona virus disease 2019: a retrospective cohort study in a designated hospital of Wuhan in China. Clin Infect Dis 2020. doi:10.1093/cid/ciaa287

18 World Health Organization. Modes of transmission of virus causing COVID-19: implications for IPC precaution recommendations. https://www.who.int/news-room/commentaries/detail/modesof-transmission-of-virus-causing-covid-19-implications-for-ipcprecaution-recommendations [Accessed on 23 May 2020]

19 Li Y, Yao L, Li J, et al. Stability issues of RT-PCR testing of SARS-CoV-2 for hospitalized patients clinically diagnosed with COVID-19. J Med Virol 2020. doi:10.1002/jmv.25786

20 Xiao AT, Tong YX, Zhang S. False-negative of RT-PCR and prolonged nucleic acid conversion in COVID-19: rather than recurrence. J Med Virol 2020. doi:10.1002/jmv.25855

21 Fan Wu AW, Mei Liu, et al. Neutralizing antibody responses to SARS-CoV-2 in a COVID-19 recovered patient cohort and their implications. medRxiv 20047365 [Preprint]. 2020. https://doi. org/10.1101/2020.03.30.20047365

22 Yong G, Yi Y, Tuantuan L, et al. Evaluation of the auxiliary diagnostic value of antibody assays for the detection of novel coronavirus (SARS-CoV-2). J Med Virol 2020. doi:10.1002/jmv.25919

23 Zhao J, Yuan $\mathrm{Q}$, Wang $\mathrm{H}$, et al. Antibody responses to SARSCoV-2 in patients of novel coronavirus disease 2019. Clin Infect Dis 2020:ciaa344. doi:10.1093/cid/ciaa344

Web appendix: Supplementary table 